\title{
Five-year outcome in immune-mediated scleritis
}

\author{
Wolfgang Bernauer • Beat Pleisch • Matthias Brunner
}

Received: 26 March 2014 / Revised: 11 June 2014 / Accepted: 12 June 2014 / Published online: 10 July 2014

(C) Springer-Verlag Berlin Heidelberg 2014

\begin{abstract}
Background Immune-mediated scleritis is a rare condition, and the information on the clinical course and complications is scarce. The aim of this study was to identify prognostic factors, complications, and therapeutic effects in patients with immune-mediated scleritis.

Methods Patients with diagnosis of scleritis and a follow-up time of 5 years were identified. Systemic disease, laboratory investigations, type of scleritis, disease activity, therapy, and complications were recorded. The study design was a retrospective, non-comparative, interventional case series.

Results Systemic disease was identified in 15 (37\%) patients at presentation and in $18(45 \%)$ after 5 years. Rheumatoid arthritis (15\%), granulomatosis with polyangiitis (7.5\%), and polychondritis $(7.5 \%)$ were the most predominant disorders. Persistent scleritis ( $>5$ years) was associated with systemic disease ( 66 vs. $6 \% ; p<0.05)$ and positive auto-antibodies (48 vs. $23 \% ; p=0.18$ ). Control of ocular inflammation was achieved in 38 of 40 (95\%). Prednisone (14 patients) and/or methotrexate (8) were the predominant drugs to control persistent disease. Complications included interstitial keratitis (2), inflammatory astigmatism (2), corneal melt (3), macular edema (6), and severe systemic disease (5).

Conclusion The presence of systemic disease and positive auto-antibodies are associated with persistent scleritis. Immunosuppressive agents allow control of scleritis, but may contribute to severe systemic complications.
\end{abstract}

This work has not previously been presented.

W. Bernauer $(\triangle) \cdot$ B. Pleisch $\cdot$ M. Brunner

OMMA Eye Center, Theaterstrasse 2, 8001 Zürich, Switzerland

e-mail: wolfgang.bernauer@hin.ch

W. Bernauer

Department of Ophthalmology, University Hospital of Zürich, Frauenklinikstrasse 24, 8091 Zürich, Switzerland
Keywords Scleritis $\cdot$ Cornea $\cdot$ Keratitis $\cdot$ Outcome . Inflammation $\cdot$ Immunopressive therapy $\cdot$ Steroids

\section{Introduction}

Scleritis often occurs as a chronic inflammatory process. Although in some instances it may be caused directly by an infective agent, the vast majority of cases present as an immune-mediated disorder [1-3]. Immune-mediated scleritis may be associated with systemic disease. Usually systemic therapy is required to control inflammation. Morphologic criteria are used to classify scleral inflammation clinically into anterior and posterior types [3]. Anterior scleritis is subclassified into diffuse, nodular, necrotising with inflammation, and necrotising without inflammation (scleromalacia).

Little information on the clinical course and complications of scleritis is available [4-7]. This study aims to gain knowledge on the long-term outcome of scleritis, and to identify prognostic factors. This was done by studying systemic disease and laboratory findings, and by the assessment of complications and therapeutic effects.

\section{Patients and methods}

In 2010 we reviewed the records of more than 100 patients who were referred to our institution with the diagnosis "scleral inflammation". Forty patients and 56 affected eyes with diagnosis of immune-mediated scleritis and the possibility to be followed by our team for the next 5 years were identified and investigated. After having obtained informed consent, these patients were included in the study. The demographic data are shown in Table 1. Immune-mediated scleritis was diagnosed on clinical grounds (constant and nocturnal pain with spreading to the periorbital region, scleral edema, tenderness of the 
Table 1 Demographic data

\begin{tabular}{ll}
\hline Age at presentation (median) & 51 years (range 31-74) \\
\hline Females/males & $65 \% / 35 \%$ \\
Relative and absolute number of patients & $100 \%(40)$ at 12 \\
at follow-up (months) & $95 \%(38)$ at 36 \\
& $95 \%(38)$ at 60 \\
& $55 \%(18)$ at 120 months \\
Deceased (relative and absolute number & $5 \%(2) 12-60$ \\
$\quad$ of cases at follow-up in months) & $17.5 \%(7) 61-120$ \\
\hline
\end{tabular}

globe, presence of nodules, or signs of scleral necrosis), and was supported in two cases by tissue examination (absence of infective agents). Patients with superficial inflammation (rather episcleritis than scleritis), infectious scleritis, neoplastic lesions, and/or incomplete data were excluded. A standardised protocol was used to record systemic disease, laboratory investigations, type of scleritis, disease activity, therapy, and complications (Table 2). The duration of scleritis was determined as abscence of clinical inflammation for more than 6 months after stopping therapy (local and systemic).

For the treatment of scleritis, an anti-inflammatory strategy was applied as published elsewhere [8]. For non-necrotising scleritis oral nonsteroidal anti-inflammatory drugs such as flurbiprofen (100-300 mg/24 h), sometimes in combination with steroids, were used to induce remission. For consolidation, methotrexate, azathioprine, anti-TNF-alpha blockers or cyclosporine were applied. Necrotising scleritis was treated with high-dose oral prednisone or intravenous methylprednisolone in combination with cyclophosphamide. Cyclophosphamide was given parenterally in an initial phase, later per os. Posterior scleritis was treated in this series solely with oral prednisone. All medication was given according to the guidelines of the Rheumatology Department, University Hospital of Zürich (Head Prof. Beat Michel) and supervised by experienced rheumatologists. Successful medical control of disease was defined as absence of symptoms, clinical inflammation and, in necrotising disease, halt of further tissue destruction.

The contingency table (see Table 6) was analysed using Fisher's exact test. Patients with persistent scleritis (more than

Table 2 Protocol for the investigation of scleritis patients

Systemic disease

Laboratory investigations (serum rheumatoid factor, ANA, ANCA)

Type of scleritis

Disease activity at presentation, $12,36,60$, and 120 months

Therapy

Complications
Table 3 Association of scleritis with systemic disease

\begin{tabular}{ll} 
At first presentation: $37.5 \%(15)$ & \\
During the further course: $45 \%(18)$ & \\
Rheumatoid arthritis & $15 \%(6)$ \\
Polychondritis & $7.5 \%(3)$ \\
Granulomatosis with polyangiitis & $7.5 \%(3)$ \\
$\quad$ (formerly Wegener's granulomatosis) & \\
Polyarteritis nodosa & $2.5 \%(1)$ \\
HLA-B27 positive (ankylosing) spondylitis & $2.5 \%(1)$ \\
HLA-B27 negative spondylitis & $2.5 \%(1)$ \\
Scleroderma & $2.5 \%(1)$ \\
Colitis ulcerosa & $2.5 \%(1)$ \\
Cogan's syndrome & $2.5 \%(1)$ \\
\hline
\end{tabular}

5 years of ongoing scleral inflammation) were compared with patients who showed a shorter course of scleral inflammation.

\section{Results}

The median age at first presentation was 51 years, and $65 \%$ were females. In $37.5 \%$ (15 patients), a systemic disorder was already known at presentation; in the further course the figure raised to $45 \%$ (18). Rheumatoid arthritis was the most frequent systemic disease (15\% or six patients), followed by polychondritis in three, and granulomatosis with polyangiitis (GPA) in three patients (Table 3). The type of scleritis at presentation is shown in Table 4: Non-necrotising anterior scleritis was the most frequent type $(77.5 \%, 31$ eyes). Necrotising anterior scleritis was seen in five and posterior inflammation in six eyes. Two eyes had both inflammation of the anterior and posterior segments. In 26 out of 40 patients, the scleritis stayed unilateral. In four patients, however, scleral inflammation became bilateral (Table 5). During follow-up, the type of scleritis changed in four patients; one patient who had initially presented with anterior disease developed posterior scleritis. One patient with initial posterior disease

Table 4 Type of scleritis at presentation

\begin{tabular}{llll}
\hline & & Unilateral & Bilateral \\
\hline Diffuse/nodular & $77.5 \%(31)$ & $45 \%(18)$ & $32.5 \%(13)$ \\
Necrotising & $12.5 \%(5)$ & $12.5 \%(5)$ & 0 \\
$\quad$ with inflammation & $7.5 \%(3)$ & $7.5 \%(3)$ & \\
$\quad$ without inflammation & $5 \%(2)$ & $5 \%(2)$ & \\
Posterior $_{\text {Total }^{\mathrm{a}}}$ & $15 \%(6)$ & $7.5 \%(3)$ & $7.5 \%(3)$ \\
\hline
\end{tabular}

${ }^{\mathrm{a}}$ Two eyes with anterior and posterior inflammation 
Table 5 Scleritis course (0-60 months)

\begin{tabular}{lr} 
Unilateral $\rightarrow$ bilateral & 4 \\
\hline Changing type of scleritis & 4 \\
Anterior $\rightarrow$ Posterior & 1 \\
Posterior $\rightarrow$ Anterior & 1 \\
Nodular/diffuse $\rightarrow$ Necrotising & 2 \\
\hline
\end{tabular}

developed non-necrotising anterior scleritis 2 years later. Two patients with non-necrotising inflammation at first visit developed ischemic areas, thus necrotising scleritis (Fig. 1).

In 21 out of 40 patients $(52 \%)$ scleral inflammation lasted more than 5 years. This persistant scleritis (more than 5 years ongoing scleral inflammation) was associated with systemic disease (14/21 in comparison to $1 / 17$ patients with shorter inflammation episodes). This association was statistically significant when analysed by Fisher's exact test. The presence of positive circulating antibodies (10/21 versus $4 / 17$ ) showed a trend to favour persistent inflammation. This is shown in Table 6 .

Information on the medical control of scleral inflammation is given in Tables 7 and 8. Control of scleritis was achieved in 38 out of 40 patients. In two patients with necrotising scleritis, the loss of scleral tissue could not be halted. One of these patients, a 75-year-old male, developed stomach carcinoma and therapy with cyclophosphamide was subsequently stopped. This patient died 18 months after first presentation. The other patient, a 39-year-old female with severe rheumatoid arthritis, developed ongoing scleromalacia despite immunosuppression with oral steroids, cyclophosphamide and TNF-alpha-blockade (Figs. 2 and 3).

The complications of scleral disease are listed in Table 9. Corneal complications included interstitial keratitis (two eyes), peripheral corneal melts (three eyes) and scleritis-induced astigmatism (two eyes) (Fig. 4, Table 10). Six patients developed cystoid macular edema. In two patients the scleritis had led to persistent structural damages and functional loss (longstanding retinal detachments without light perception). Several patients developed severe systemic disease: myocardial

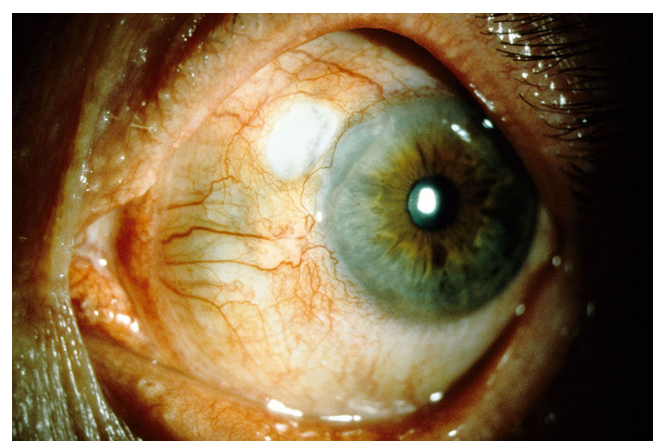

Fig. 1 patient with non-necrotising at first visit developed ischemic areas, thus necrotising scleritis
Table 6 Duration of scleritis

\begin{tabular}{llll}
\hline & $<5$ years & $>5$ years & $p$ value* \\
\hline Percentage (effective) & $43 \%(17)$ & $52.5 \%(21)$ & \\
Systemic disease & $1 / 17$ & $14 / 21$ & $<0.05$ \\
Autoantibodies & $4 / 17$ & $10 / 21$ & 0.18 \\
$\quad$ Serum rheumatoid factor & 2 & 7 & \\
$\quad$ (ELISA $>25 \mathrm{mu} / \mathrm{ml})$ & & & \\
ANA (Titer, $>1: 10)$ & 2 & 5 & \\
ANCA (Titer, $>1: 10)$ & 0 & 3 & \\
\hline
\end{tabular}

No clinical inflammation for $>6$ months after stopping therapy (local and systemic)

* $p$ by Fisher's exact test

infarction at age 44 and IgA-nephropathy, pericarditis with valvular and coronary heart disease (possibly associated with the polyarteritis nodosa), and two patients died within the 5year follow-up period (Table 11).

\section{Discussion}

Information on the outcome of scleritis is scarce. To date, there are case reports and the Moorfields, Boston, and Baltimore cohorts $[1,3,6]$ that help to understand the nature of scleral inflammation. Our study sets the focus on the long-term course of scleritis and, in this way, teaches us about the outcome of immune-mediated scleritis.

Scleritis is a potentially long-lasting problem. Fifty-two percent of our patients showed a disease duration of more than 5 years. Although this figure may be biased by the fact that we see patients as tertiary referrals, we were surprised by this high number. An underlying systemic disease and positive autoantibodies were identified as risk factors for such a long disease duration.

Melts, interstitial keratitis, and peripheral ulcerative keratitis are well known corneal complications of scleritis [4-7]. Inflammatory astigmatism due to scleritis was previously described $[9,10]$, but none of those patients had such a high cylinder measurement as our patient with 8.5 diopters (Table 10). A second patient of our series had a cylinder measurement of 2.5 diopters. In both patients it was reversible after remission of scleritis. We feel that it is the increase in

Table 7 Medical control of disease activity

\begin{tabular}{lll}
\hline & Yes & No \\
\hline Control of inflammation & 38 & $2^{\mathrm{a}}$ \\
\hline
\end{tabular}

No discomfort, no further destruction

${ }^{\text {a }}$ Ongoing destruction in the two patients with scleromalacia 
Table 8 Systemic medication in 40 scleritis patients

\begin{tabular}{|c|c|c|c|}
\hline & 0-1 months & 12 months & 36 months \\
\hline NSAID & 20 & 10 & 3 \\
\hline Prednisone & 21 & 22 & 14 \\
\hline Cyclophosphamide & 5 & 3 & - \\
\hline Methotrexate & 5 & 6 & 8 \\
\hline Azathioprine & 2 & 1 & 1 \\
\hline \multicolumn{4}{|l|}{ Anti-TNF-alpha } \\
\hline Infliximab (Remicade $\left.{ }^{\circledR}\right)$ & & & 1 \\
\hline Adalimumab (Humira $\left.{ }^{\circledR}\right)$ & & 1 & 1 \\
\hline Cyclosporine A & 1 & 1 & \\
\hline
\end{tabular}

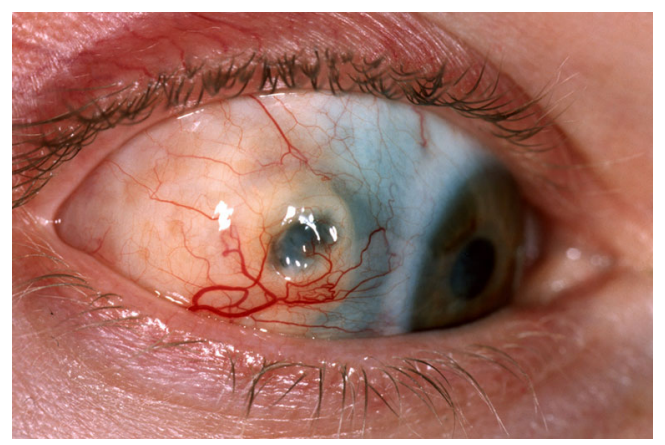

Fig. 2 Scleromalacia in a 39-year-old female with severe rheumatoid arthritis. There is progressive scleral destruction despite immunosuppression with oral steroids, cyclophosphamide, and TNF-alpha blockade

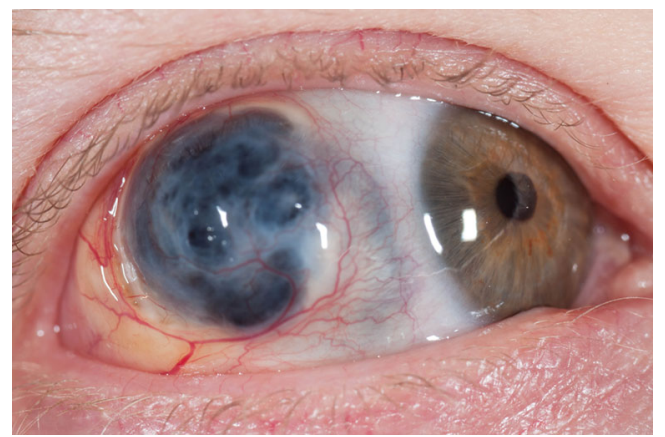

Fig. 3 The same eye as in Fig. 2. The progression over a period of 5 years is obvious
Table 9 Complications (months 0-60)

$\begin{array}{ll}\text { Interstitial keratitis } & 2 \\ \text { Scleritis-induced astigmatism } & 2 \\ \text { Peripheral corneal melt } & 3 \\ \text { Cystoid macular edema } & 6 \\ \text { Persistent structural damage } \rightarrow \text { enucleation } & 2 \\ \text { Severe systemic disease } & 5 \\ \quad \text { Myocardial infarction at age 44, IgA-nephropathy } & 1 \\ \text { Pericarditis, valvular/coronary heart disease } & 1 \\ \text { Deceased (patients \#5 and \#6)* } & 2\end{array}$

*For details of these patients see Table 11

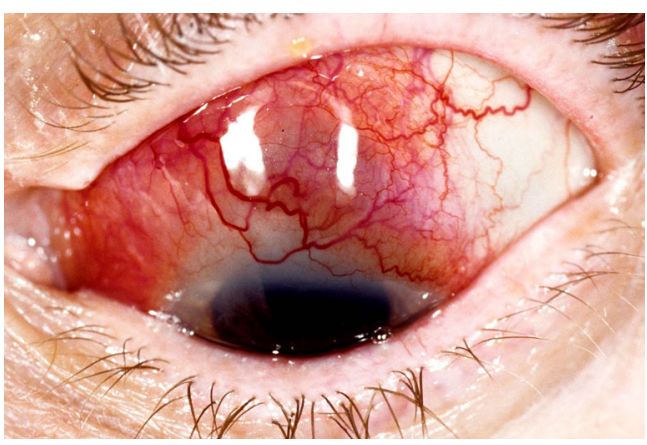

Fig. 4 A 73-year-old female who developed anterior and posterior nonnecrotizing scleritis. The scleral nodule in the upper and nasal quadrants resulted in a corneal astigmatism of -8.50 in $117^{\circ}$. The course of the corneal curvature is shown in Table 10

Table 10 Corneal astigmatism before and after onset of anterior nonnecrotizing scleritis which responded to treatment with oral steroids. The affected eye is shown in Fig. 4

\begin{tabular}{ll}
\hline Follow-up time & Autorefraction (NIDEK ARK-700A) \\
\hline Pre-inflammatory refraction & $-3.50-1.25110^{\circ}$ \\
Day 1 & $\mathrm{a}-8.50117^{\circ}$ \\
Day 14 & $-1.00-6.00110^{\circ}$ \\
Day 28 & $-1.75-4.50116^{\circ}$ \\
Day 42 & $-1.50-4.25113^{\circ}$ \\
Day 90 & $-0.75-3.75111^{\circ}$ \\
Day 120 & $-1.00-3.75111^{\circ}$ \\
\hline
\end{tabular}

${ }^{\mathrm{a}}$ Sphere not measurable 
Table 11 Deaths (0-120 months)

\begin{tabular}{|c|c|c|c|c|}
\hline Patient & Cause of death & $\begin{array}{l}\text { Type and duration } \\
\text { of scleritis (months) }\end{array}$ & $\begin{array}{l}\text { Systemic } \\
\text { inflammatory disease }\end{array}$ & Systemic medication \\
\hline C.A., $\widehat{O}$, age 76 years, \# 5 & Stomach carcinoma & Necrotising, 9 & & Steroids, cyclophosphamide \\
\hline E.M.,, , age 68 years, \#6 & Not known & Anterior and posterior, $>60$ & & Steroids, azathioprine \\
\hline E.W., ठ̊, age 72 years, \# 7 & Aplastic anemia & Necrotising, 38 & Rheumatoid arthritis & $\begin{array}{l}\text { Steroids, methotrexate, } \\
\text { cyclophosphamide, } \\
\text { later azathioprine }\end{array}$ \\
\hline H.HR., ô, age 45 years, \# 12 & Sepsis (immunosuppression?) & Diffuse anterior, 120 & Polychondritis & Steroids, infliximab \\
\hline K. H., ô, age 70 years, $\# 13$ & Renal failure & Diffuse anterior, 21 & Polychondritis & Steoids, cyclosporine \\
\hline L. M.,, , age 80 years, \# 17 & Heart failure & Diffuse anterior, 15 & Cogan's syndrome & Steroids, cyclophosphamide \\
\hline S.F., ô, age 79 years, \# 26 & Aortic aneurysm & Necrotising, 9 & & Steroids, cyclophosphamide \\
\hline
\end{tabular}

volume of the inflamed sclera that leads to changes of scleral rigidity with subsequent instability of limbal contour and corneal steepening in the affected quadrant.

Macular edema was observed in $11 \%$ of our patients. When detected at an early stage it was fully reversible after high-dose oral steroids. One of our patients presented with posterior scleritis only and developed anterior scleritis years later in the same eye, and three other eyes changed the type of scleritis (Table 5). This is in contrast to Tuft's study [11] who found that the majority of patients remain in the same clinical category. It seems that a change in the scleritis pattern is not unusual when patients are available for long-term follow-up.

The evaluation of the best medication for the treatment of scleritis was beyond the scope of this study. With the correct application of traditional and new generation immunosuppressive agents, preferably in collaboration with a rheumatologist, disease control is possible in most cases. In our series, remission was achieved in $95 \%$. In one patient with long-standing rheumatoid arthritis and scleromalacia (Figs. 2 and 3), tissue loss could not be halted despite application of TNF-alpha blockers and, at a later stage, cyclophosphamide in combination with oral steroids. This demonstrates again that this vasculitic complication is particularly difficult to tackle $[1,6,7]$.

Our patients showed a considerable morbidity and a high number of lethal complications. Five percent of our patients died within 5 years and $17.5 \%$ within 10 years of follow-up time. Three of the deaths were older than 75 years and presumably died of natural causes. Of concern are the two patients who died with aplastic anemia and septicemia. This demonstrates that the immunosuppressive therapies have the potential to cause lethal complications, although the systemic disease itself may be life-threatening $[1,2]$. New agents allow control of scleritis, but may also contribute to systemic complications.

Proprietary interest None.

\section{References}

1. Akpek EK, Thorne JE, Qazi FA, Do DV, Jabs DA (2004) Evaluation of patients with scleritis for systemic disease. Ophthalmology 111: 501-506

2. Sainz de la Maza M, Foster CS, Jabbur NS (1995) Scleritis associated with systemic vasculitic diseases. Ophthalmology 102:687-692

3. Watson PG, Heyreh SS (1976) Scleritis and episcleritis. Br J Ophthalmol 60:163-191

4. Bernauer W, Ficker LA, Watson PG, Dart JKG (1995) The management of corneal perforations associated with rheumatoid arthritis. Ophthalmology 102:1325-1337

5. Sainz del la Maza, Jabbur NS, Foster CS (1993) An analysis of therapeutic decisions for scleritis. Ophthalmology 100:1372-1376

6. Sainz de la Maza M, Molina N, Gonzalez-Gonzalez LA, Doctor PP, Tauber J, Foster CS (2012) Clinical characteristics of a large cohort of patients with scleritis and episcleritis. Ophthalmology 119:43.50

7. Wieringa WG, Sieringa JE, Dam-van Loon NH, Los LI (2013) Visual outcome, treatment results, and prognostic factors in patients with scleritis. Ophthalmology 120:379-386

8. McCluskey PJ, Wakefield D (1996) Scleritis and Episcleritis. In: Pepose JS, Holland GN, Wilhelmus KR (eds) Ocular infection and immunity, 1st edn. Mosby, St. Louis, pp 642-662

9. Arellanes-Garcia L, del Carmen Preciado-Delgadillo M, Graza-Leon M (2011) Refractive changes in patients with autoimmune scleritis. J Ophthalmol Inflamm Infect 1:173-175

10. Meller D, Böker T (1997) Complications of misdiagnosed and inadequately treated necrotizing scleritis studied by ultrasound biomicroscopy and computerized corneal topography. Int Ophthalmol 21(1):35-37

11. Tuft SJ, Watson PG (1991) Progression of scleral disease. Ophthalmology 98:467-471 